\title{
On Public Safety LTE Network: Simulation Results in Uplink From a Video Surveillance Use Case
}

\author{
Eric Galloix \\ Nokia Networks, Oulu, Finland
}

\begin{abstract}
This paper presents a study on uplink capacity of an LTE public safety network supporting video surveillance in order to assist first responders when they need to visualize remotely a critical area. Using system-level simulations, the number of supported surveillance camera users within a cell area of interest is determined. Targeted bit rate in uplink is $512 \mathrm{kbps}$.
\end{abstract}

\section{INTRODUCTION}

Broadband wireless access (BWA) networks like 3GPP Long Term Evolution (LTE) [1] or upcoming 5G (though not yet standardized) are becoming important to help first responders, for example governmental organizations, to accomplish their missions of security [2], especially when they have to transmit emergency video data, acting as uplink users in the network. Private Mobile Radio (PMR) technologies have reached their limits against current needs [2], and that is mainly why the regulator and public safety standardization organizations in the US have selected LTE technology. Therefore in this paper we study with system-level simulations the capacity in uplink of a specific LTE video surveillance system, where the number of supported surveillance camera users within a cell area of interest will be determined, for example at the location of an accident. In our study, video surveillance cameras have low mobility and send data continuously at a bit rate of $512 \mathrm{kbps}$. Intra and inter cell interferences as well as interferences between users are taken into consideration. This case-study implies that the public safety network of base stations should be independent from the telecom operator networks because video surveillance operations should remain invisible to ordinary or legacy end-users. A commercial LTE network could be used as well for this purpose if appropriate sharing or pre-emption mechanisms are implemented. Also relying on a single private network may not be robust under, for example, nature disasters where infrastructure may be at risk.

In this context, we concentrate on video service in uplink direction, motivated by the growing potential for 3GPP-based mobile broadband technologies (LTE, 5G) to deliver such services with increasing spectral efficiency, higher bandwidth availability, and enhanced air interface solutions [3].

Beside Quality of Service (QoS) requirements, it is worth mentioning the existence of subjective Quality of Experience (QoE) measures containing at least three objectives [4] related to video streaming. As long term average video quality is of importance in video surveillance, it motivates our study to detect users in outage situation. The scale of visual quality change can be controlled using guaranteed bit rate (GBR) parameter, whose maximum value depends on the performance of the available Internet Protocol (IP) backhauls. The third objective which is playback jitter also known as delay variation is not applicable in our study because we assume constant latency, the variance of the packet delay variation is zero. The targeted supported bit rate $512 \mathrm{kbps}$ can be drawn from the IP network point of view, recalling that an LTE (or 5G) core network is built on IP backhaul. Considering IPv4 with TCP services where the packet size is typically 1468 bytes with robust header compression [5], and assuming as an example a packet interval of $20 \mathrm{~ms}$ as in Voice over IP (VoIP), the available bit rate is $587 \mathrm{kbps}$.

In Section II, we review similar case-studies from the literature. Simulation parameters and results are presented respectively in Section III and Section IV. A conclusion is drawn in Section V.

\section{RELATED WORK}

Performance results of policy-based resource allocation for incident scene management were reported via simulations in the literature [6]. In the same shared network, mission-critical users were given more bandwidth upon request than nonmission-critical sessions according to predefined policy rules drawn from QoS requirements. However neither the system layout nor the wireless system technology was documented.

Simulation results for a constant bit rate (CBR) of $250 \mathrm{kbps}$ from wireless personal area networks (WPAN) with multimedia sensors were reported [7], where the communication range barely exceeds 10 meters, whereas our aim is to run LTE intersite system simulations with a distance of 500 meters.

Reliable transmission of video surveillance streams has also been studied [8]. Reliability in video surveillance means that not even a single image can be lost, which is a more stringent requirement than a threshold-based packet loss. In this respect, a solution cascading Transmission Control Protocol (TCP), a multicast video server and User Datagram Protocol (UDP) was proposed in order to provide several viewers with the captured streams from a wireless IP camera. However they assumed that the video was taken for a long period of time with period of activity and inactivity and rare background changes, whereas we prefer to place ourselves in the worst case scenario with possible sudden illumination changes in outdoor conditions or rapidly moving objects. In [8] authors provide numerical results on capacity over IEEE $802.11 \mathrm{~b}$ networks, and we would like to propose the same kind of results for 3GPP LTE 
networks though we will use an LTE system simulator with an appropriate traffic model.

In the downlink direction, a resource allocation method targeted to video streaming has been proposed in [9], but we are concentrating on the uplink direction. Live and on-demand LTE uplink video transmission has also been investigated in [10] for a fixed amount of three users, whereas our study focuses on measuring the capacity when interferences from neighbour cells are taken into account.

\section{SyStem-LEVEL Simulations}

\section{A. Simulation Model}

The LTE system simulator [11] has a granularity of one physical resource block (PRB) [1]. An information symbol $x_{u}$, a complex number, is transmitted every subframe $(1 \mathrm{~ms})$ from a user equipment (UE) of index $u$ to an eNB's sector. The received signal complex vector $\overrightarrow{y_{u}}$ at the same eNB's sector is modeled in (1), where the length of $\overrightarrow{y_{u}}$ is $N_{r}$ that is the number of receive antennas. The channel from the UE $u$ to the eNB's sector is $H_{u}$. The background white noise has a standard deviation $\sigma$. The first term in (1) stands for the desired signal, the second term for the inter-cell interference and the last term is for the noise contribution.

$$
\overrightarrow{y_{u}}=H_{u} x_{u}+\sum_{j \neq u} H_{j} x_{j}+\sigma \vec{I}
$$

The estimated input $\widehat{x_{u}}$ at the receiver is given by (2), where $\overrightarrow{z_{u}}$ is calculated using (3) which is a minimum mean square estimator, and where the star stands for the complex conjugate. The channel estimate $\widehat{H_{u}}$ in (4) depends on the channel estimation error based on a dedicated reference signal and the distribution of signal-to-interference-plus-noise ratio (SINR) in the area of interest, including also Doppler effects. An estimated covariance model $\widehat{X_{u}}$ is given in (5) using intercell interferences.

$$
\begin{gathered}
\widehat{x_{u}}=\overrightarrow{z_{u}} \vec{y}_{u} \\
\widehat{z_{u}}={\widehat{H_{u}}}^{\star}\left(\widehat{H_{u}}{\widehat{H_{u}}}^{\star}+\widehat{X_{u}}\right)^{-1} \\
\widehat{H_{u}}=H_{u}+\vec{e} \\
\widehat{X_{u}}=\sum_{j \neq u} \widehat{H}_{j} \widehat{H}_{j}^{\star}+\sigma^{2} \vec{I}
\end{gathered}
$$

Power control in uplink direction [12] has been standardized by $3 \mathrm{GPP}$ for LTE, e.g. for the open-loop as in (6) in $\mathrm{dBm}$ unit, where $P_{\max }$ is the maximum transmit power for a user, $P_{0}$ is the initial power, $\mathrm{M}$ is the number of PRB's allocated to the considered user, $\alpha$ is a path-loss compensation factor, and $L$ is the downlink path-loss seen from the UE measured using reference symbols. When $\alpha=1$ for all of the users (full compensation of the path loss) the eNB decodes all the users with the same SINR. In closed-loop, the formula is adjusted based on the selection of a modulation and coding scheme (MCS) and on the received scheduling grant indicating a power

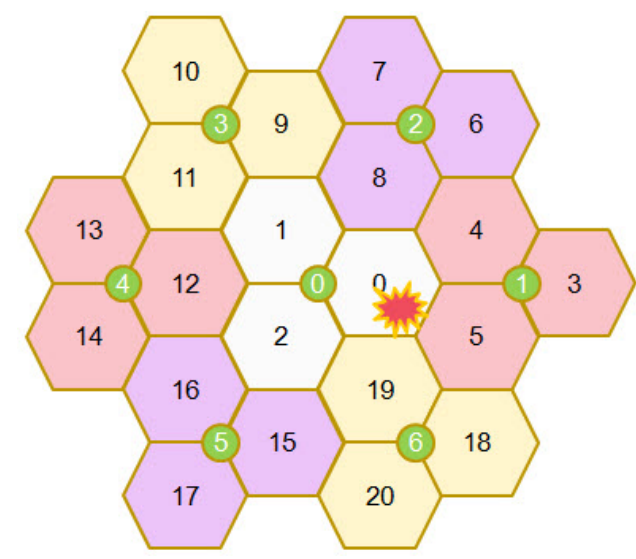

Fig. 1. System layout with cell 0 as the cell of interest

adjustment. The equation (6) gives the power of the physical uplink shared channel (PUSCH) applied at a subframe $s$ :

$$
P_{s}=\min \left(P_{\max }, P_{0}+10 \log _{10} M+\alpha L\right)
$$

A link-to-system table contains block-error rate (BLER) values versus SINR values for each selected MCS used by the PUSCH channel.

The selected traffic model has an infinitely full buffer, we can simulate mobiles that have always data available to be sent in uplink direction at each time transmission interval of $1 \mathrm{~ms}$ (TTI).

\section{B. Simulation parameters}

Placed in an hexagonal layout, the number of base station sites is 7 , each of them covering 3 sectors, providing the system with 21 cells. The cell numbered 0 in site 0 is at the centre and is assumed to be the site where an incident is detected. The remaining adjacent sites are numbered from 1 to 6 in an anti-clockwise direction around the centre as depicted in Fig. 1. The cells are numbered in the same manner.

The LTE bandwidth of the considered system simulator is 10 $\mathrm{MHz}$ in frequency-division duplexing (FDD) mode operating at a carrier frequency of $2 \mathrm{GHz}$. 3GPP macro-cell case 1 [13] is selected as a system simulation scenario where user equipment speed is $3 \mathrm{~km} / \mathrm{h}$ and inter-site distance 500 meters. Video surveillance is usually static but in order to slightly consider Doppler effects in the simulation, this scenario is suitable. A static UE, meaning a speed of zero $\mathrm{km} / \mathrm{h}$, would imply disabling Doppler effects from the simulation model, consequently that would not be a realistic enough scenario and that would require much more simulation samples over time.

The number of Physical Resource Blocks (PRBs) per user may vary from 1 to 48 according to channel conditions, the maximum being 50 PRBs for a bandwidth of $10 \mathrm{MHz}, 2$ PRB's being allocated for the physical uplink control channels (PUCCH). Also the UE's sending more frequently at maximum power gets a smaller PRB allocation. The available MCS indexes are mapped in ascending order to QPSK $1 / 10$, $1 / 6,1 / 4,1 / 3,1 / 2,2 / 3,3 / 4,16$ QAM $1 / 2,2 / 3,3 / 4,5 / 6$ and 


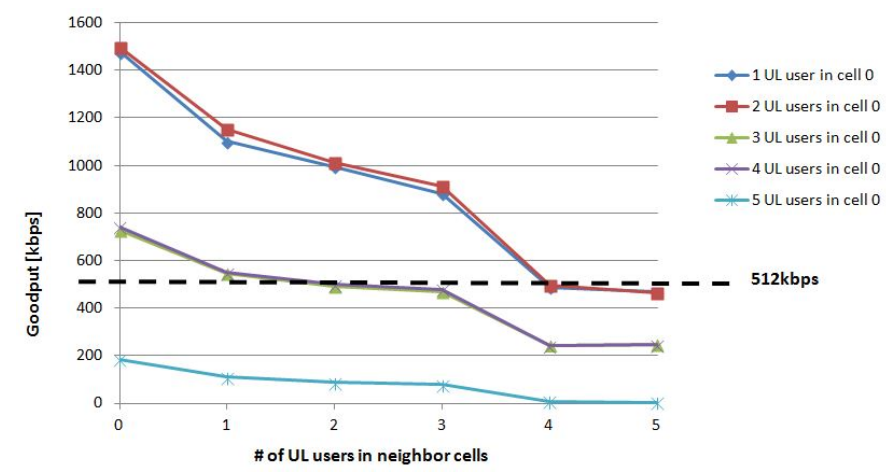

Fig. 2. User goodput at cell edge in cell 0 versus the number of UL camera users in neighbour cells

64 QAM 2/3,3/4, 5/6, 8/9. Adaptive modulation and coding scheme (MCS) selects a higher coding rate or redundance, e.g. at cell edge when a user needs to adapt to a variable level of interferences. Proportional fair scheduling (PFS) is chosen with a fairness factor set to 1 , because PFS takes into account the latency for each user independently of the channel state. Channel estimation is non-ideal on typical urban channel model. All UE's are assumed to be placed in outdoor conditions, i.e. a penetration loss of $0 \mathrm{~dB}$. $P_{0}$ is set to -81 $\mathrm{dBm}$, and $\alpha=0.8$. The UE transmission power is $23 \mathrm{dBm}$. Thermal noise level is set to $-174 \mathrm{dBm} / \mathrm{Hz}$. The number of receive antennas $N_{r}$ is 2 , and the UE has only one transmit antenna. A minimum of fifty simulation runs of duration 1 seconds is used to get goodput results for sufficiently different UE locations.

\section{Simulation Results}

In this section, we look at outage results for uplink users in the cell 0 where an incident has been detected. The curves reported in Fig. 2 shows that up to 4 surveillance cameras can still operate at $512 \mathrm{kbps}$ in cell 0 when there are 2 users in neighbour cells. Another possible setup is with up to 2 surveillance cameras that can operate at $512 \mathrm{kbps}$ when there are 4 users in neighbour cells.

As a comparison, 10 users were used in [7] (mentioned in Section II) in a single cell context for a CBR $(250 \mathrm{kbps})$ twice lower than the constraint set in this study. From the results presented in Table 1 of [8] for H.264 video over $802.11 b$ inside a single cell, i.e. without inter-cell interferences, it can be seen that the data rate for up to 4 surveillance cameras is $1241 \mathrm{kbps}$, which is nearly twice higher than the constraint set in this study.

\section{CONCLUSION}

In this paper, we determined using system-level simulations the number of LTE wireless video surveillance cameras that can simultaneously visualize and transmit a scene of interest at a bit rate of $512 \mathrm{kbps}$ while considering interferences created by neighbour cells and users.
The topic treated in this paper rejoins Machine Type Communication (MTC) [14] which appears in 3GPP standardization bodies (potential extension to $5 \mathrm{G}$ ), where e.g grouping of sensors is addressed. But usually in MTC the devices are not continuously sending data, rather they are assumed to work with small packet size and to transmit data less frequently than digital video surveillance cameras.

Also in order to have a more realistic model for the simulations in this paper, a finite buffer model, for example users operating at GBR of $512 \mathrm{kbps}$, could be used instead of the selected full buffer approach which does not set any constraints on user throughput.

\section{REFERENCES}

[1] C. Johnson, Long Term Evolution in bullets. CreateSpace Independent Publishing Platform, 2010.

[2] J. Brouet and S. Feng, "Lte and future evolutions for the benefits of security wireless networks," in IET International Communication Conference on Wireless Mobile and Computing, 2011, pp. 447-554.

[3] O. Oyman, J. Foerster, Y. Tcha, and S. Lee, "Toward enhanced mobile video services over wimax and lte," IEEE Communications Magazine, pp. 68-76, Aug. 2010.

[4] W. Pu, Z. Zou, and C. W. Chen, "Video adaptation proxy for wireless dynamic adaptive streaming over http," in 19th IEEE International Packet Video Workshop (PV), 2012.

[5] A. Larmo, M. Lindstrom, M. Meyer, G. Pelletier, J. Torsner, and H. Wiemann, "The lte link-layer design," IEEE Communications Magazine, pp. 52-59, Apr. 2009.

[6] J. Q. Bao, L. Guo, and W. C. Lee, "Policy-based resource allocation in a wireless public safety network for incident scene management," in DIWANS '06: Proceedings of the 2006 workshop on Dependability issues in wireless ad hoc networks and sensor networks, 2006.

[7] S. Persia and D. Cassioli, "Ipv4wireless multimedia sensor networks," in Third International Workshop on Software Engineering for Sensor Network Applications (SESENA), 2012, pp. 58-63.

[8] F. Andritsopoulos, S. Papastefanos, G. Georgakarakos, and G. Doumenis, "Reliable multicast h.264 video streaming for surveillance applications," in IEEE 18th International Symposium on Personal, Indoor and Mobile Radio Communications(PIMRC), 2007, pp. 1-4.

[9] H. A. M. Ramli, K. Sandrasegaran, R. Basukala, R. Patachaianand, M. Xue, and C.-C. Lin, "Resource allocation technique for video streaming applications in the lte system," in 19th Annual Wireless and Optical Communications Conference (WOCC), 2010, pp. 1-5.

[10] A. E. Essaili, L. Zhou, D. Schroeder, E. Steinbach, and W. Kellerer, "Qoe-driven live and on-demand lte uplink video transmission," in IEEE 13th International Workshop on Multimedia Signal Processing (MMSP), 2011, pp. 1-6.

[11] F. D. Calabrese, C. Rosa, M. Anas, P. Michaelsen, K. Pedersen, and P. Mogensen, "Adaptive transmission bandwidth based packet scheduling for lte uplink," in IEEE 68th Vehicular Technology Conference, 2008, pp. $1-5$.

[12] A. Simonsson and A. Furuskar, "Uplink power control in lte - overview and performance, principles and benefits of utilizing rather than compensating for sinr variations," in IEEE 68th Vehicular Technology Conference, 2008, pp. 1-5.

[13] Physical layer aspect for evolved Universal Terrestrial Radio Access (UTRA), 3GPP TR 25.814, Rev. 7.0.0.

[14] Service Requirements for Machine-Type Communications, 3GPP TS 22.368, Rev. 11.0.0, 2010. 